TRANSACTIONS OF THE

AMERICAN MATHEMATICAL SOCIETY

Volume 236, February 1978

\title{
THE METABELIAN $p$-GROUPS OF MAXIMAL CLASS
}

BY

R. J. MIECH

\begin{abstract}
This paper presents a solution to the isomorphism problem for the set of metabelian p-groups of maximal class.
\end{abstract}

Several terms need to be defined. Let $G$ be a group, $G_{2}=[G, G]$ be the commutator subgroup of $G$, and $G_{i+1}=\left[G_{i}, G\right]$ for $i>2$. Then $G$, of order $p^{n}$, is of maximal class if $\left|G: G_{2}\right|=p^{2}$ and $\left|G_{i}: G_{i+1}\right|=p$ for $i=2, \ldots, n$ - 1. If $G$ is of maximal class then, by definition, $G_{1}$ is the largest subgroup of $G$ such that $\left[G_{1}, G_{2}\right] \leqslant G_{4}$. It is known $\left[3\right.$, p. 361] that $G_{1}$ is a characteristic subgroup of index $p$ in $G$ and there is a unique integer $k>4$ such that $\left[G_{1}, G_{2}\right]=G_{k}$. The basic facts about these groups can be found in [1] or [3].

The motivation for the study of the present problem grew out of an earlier work [4] where the metabelian $p$-groups of maximal class and order $p^{n}$ with $n>2 p$ were classified. Under the hypothesis $n>2 p-2$ the class of $G_{1}$ is at most 2 so one can get a hold on the problem by explicit calculation of several commutators.

The commutator calculation method fails in the small order case, for if $n<p+1$ the class of $G_{1}$ may be as large as $(n-1) / 2$. The solution presented in this paper is based on the fact that $G$ is generated by two elements $x$ and $y$, which may be considered as abelian operators on $G_{2}$, and one of them, say $y$, is a polynomial in $x$. Exploitation of this polynomial relation enables one to avoid commutator calculations.

The difference between the case considered in [4] and the one considered here may also be illustrated in slightly more technical terms. In [4] the isomorphism problem reduces to a set of equations that are linear in terms of a set of parameters $a(k), \ldots, a(n-1)$. In this paper the reduction is to a set of equations that are linear in terms of the summatory functions, $A(m, n)$, of these parameters. That is, functions of the form

$$
A(m, n)=\sum_{x_{1}, \ldots, x_{m}} a\left(x_{1}\right) \cdots a\left(x_{m}\right)
$$

where the summation is over those $\left(x_{1}, \ldots, x_{m}\right)$ with $x_{1}+\cdots+x_{m}=n+$

Received by the editors February 6, 1976.

AMS (MOS) subject classifications (1970). Primary 20D15; Secondary $20 \mathrm{D} 45$. 
$2 m$. The general problem is, consequently, somewhat more complicated than the large order case.

We begin with a descriptive result:

THEOREM 1. Let $p$ be a prime with $p>5$. Let $G$ be a metabelian $p$-group of maximal class and order $p^{n}$ where $n>5$. Then there is a set of generators $\{x, y\}$ of $G, a$ basis $\left\{u_{2}, \ldots, u_{n-1}\right\}$ of $G_{2}$, an integer $k$, and a set of integers $\{a(k), \ldots, a(n-1), w, z\}$ with $a(k) \neq 0 \bmod p$ such that $G_{1}=\left\langle y, G_{2}\right\rangle$ and

$$
\begin{aligned}
& {[y, x]=u_{2},\left[u_{i}, x\right]=u_{i+1} \text { for } i>1 \text {, }} \\
& {\left[u_{2}, y\right]=u_{k}^{a(k)} \cdots u_{n-1}^{a(n-1)} \text {, }} \\
& x^{p}=u_{n-1}^{w}, y^{p} u_{2}\left(\begin{array}{l}
p \\
2
\end{array}\right) \cdots u_{p}^{\left(\begin{array}{c}
p \\
p
\end{array}\right)}=u_{n-1}^{2},
\end{aligned}
$$

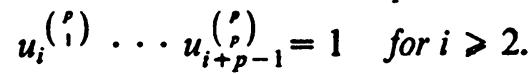

The integer $k$ is an invariant of the group. If $n>p+2$ then $k>n-p+2$; if $n<p+1$ then $k>4$. Finally, if $c$ is the nilpotence class of $G_{1}$ then $c=[(n-$ $3) /(k-2)]+1$.

Several comments about Theorem 1 are in order. First, it will be taken as known; it can be proved by several applications of the theorem on cyclic extensions. Second, we shall not consider the classification for the cases $p=2, p=3,|G|<p^{4}$, or $G_{1}$ is abelian. The first three can be found in [3, p. 362]; the last is discussed in [1, p. 88].

Next, since $[g, x]=g^{x-1}$ for $g \in G_{2}$, and since $G$ is metabelian, we may consider $x$ and $y$ as abelian operators on $G_{2}$. Strictly speaking we are then working with the ring of polynomials in two variables with integral coefficients modulo the ideal defined by:

$$
\begin{gathered}
(x-1)^{n-2}=0, \quad \sum_{i=1}^{p}\left(\begin{array}{l}
p \\
i
\end{array}\right)(x-1)^{i-1}=0, \\
(y-1)=\sum_{i=k}^{n-3} a(i)(x-1)^{i-2} .
\end{gathered}
$$

However, by (iv), $G_{2}$ is of exponent $p$ if $n<p+1$ and $G_{n-p+1}$ is of exponent $p$ when $n>p+2$. Thus, since $k>4$ or $k>n-p+2$, the parameters $a(k), \ldots, a(n-1), w$, and $z$ may be considered as residues modulo $p$.

Finally, the statement in Theorem 1 about the nilpotence class of $G_{1}$ follows directly from the above relation for $(y-1)$.

The groups of Theorem 1 will be split into disjoint classes in the following way: Let $k$ be the invariant mentioned in Theorem 1 . Let $h$ be a nonnegative integer and $q(0), \ldots, q(h)$ be a sequence of integers with $k=q(0)<q(1)$ $<\cdots<q(h)<n-1$ and $q(i) \neq 2 k-3$ for all $i$. 
The $q(i) \neq 2 k-3$ condition is vacuous when $2 k-3>n$. In particular, it is vacuous when $n>2 p-1$ since $k>n-p+2$. Note also that if $2 k-3$ $>n$ then there are $2^{n-1-k}$ sequences $q(0), \ldots, q(h)$; if $2 k-3<n-1$ there are $2^{n-2-k}$.

To continue, suppose $h$ and $q(0), \ldots, q(h)$ are fixed. Let $\left(a_{0}(q(0)), \ldots\right.$, $\left.a_{h}(q(h))\right)$ be a $(h+1)$-tuple of nonzero residues modulo $p$. Let $P(\nu)=$ $a_{0}(q(0)) \cdots a_{v}(q(\nu))$ for $\nu=0, \ldots, h$. Let $S_{n}^{m}$ be a Stirling number of the first kind. It is defined by

$$
(x)_{n}=x(x-1) \cdots(x-n+1)=\sum_{m=0}^{n} S_{n}^{m} x^{m} .
$$

By convention, $S_{n}^{m}=0$ when $m>n$. Let, for $i=k, \ldots, n-1$,

(1) $a(i)=\sum_{j=1}^{n} \frac{1}{j !} \sum_{\nu_{1}=0}^{n} \cdots \sum_{\nu_{j}=0}^{n} P\left(\nu_{1}\right) \cdots P\left(\nu_{h}\right) \frac{(Q(j)-2 j) !}{(i-2) !} S_{i-2}^{Q(j)-2 j}$,

where $Q(j)=Q\left(\nu_{1}, \ldots, v_{j}\right)=q\left(\nu_{1}\right)+\cdots+q\left(\nu_{j}\right)$. Let $C(q(0), \ldots, q(h))$ be the set of those groups in Theorem 1 whose parameters $a(k), \ldots, a(n-$ 1) are defined by (1). Since any group in this class is defined in terms of $a_{0}(q(0)), \ldots, a_{h}(q(h)), w$, and $z, \mathcal{C}(q(0), \ldots, q(h))$ contains at most $(p-$ 1) ${ }^{h+1} p^{2}$ groups.

The multiple sum in (1) reduces to

$$
a(i)=\sum_{\nu=0}^{h} P(\nu) \frac{(q(\nu)-2) !}{(i-2) !} S q_{-2}^{(\nu)-2}
$$

when $i<2 k-3$. For $S_{i-2} Q(j)-2 j=0$ when $Q(j)-2 j>i-2$ and $Q(j)=$ $q\left(\nu_{1}\right)+\cdots+q\left(\nu_{j}\right)>k j$. In particular, all the multiple sums reduce to a single sum when $2 k-3>n-1$; this is always the case when $n>2 p-2$. Note also that $a(k)=a_{0}(q(0))$.

We shall see that each group in Theorem 1 belongs to a unique class $\mathcal{C}(q(0), \ldots, q(h))$, if $G$ is in $\mathcal{C}(q(0), \ldots, q(h))$ then $\{q(0), \ldots, q(h)\}$ is a set of invariants for $G$, and groups from different classes are not isomorphic. The isomorphism question within each class is answered by

THEOREM 2. Suppose $G$ and $\bar{G}$ are in $\mathcal{C}(q(0), \ldots, q(h)), G$ is defined by $\left\{a_{0}(q(0)), \ldots, a_{h}(q(h)), w, z\right\}, \bar{G}$ is defined by $\left\{\bar{a}_{0}(q(0)), \ldots, \bar{a}_{h}(q(h)), \bar{w}, \bar{z}\right\}$ and $G$ is isomorphic to $\bar{G}$. Then there is an isomorphism $\vartheta$ from $\bar{G}$ to $G$ that is defined by $\bar{x}^{\vartheta}=x^{\alpha} y^{\beta}$ and $\bar{y}^{\vartheta}=y^{\delta}$ where $(\alpha \delta, p)=1$. In addition, 


$$
\delta a(k)=\alpha^{k-2} \bar{a}(k),
$$

(iii) $z=\alpha^{n-2}[\bar{z}-\bar{\psi} 2(\beta / \delta)], \quad w=\alpha^{n-3}\left[\bar{w} \delta-\bar{z} \beta+\bar{\psi}\left(\beta^{2} / \delta\right)\right]$,

where $\bar{\psi}=\bar{a}(k)$ if $k=n-p+2$ and $\bar{\psi}=0$ otherwise. Finally, if $2 k-3<n$ -1 then $\beta=0$.

The equations in (i) and (ii) come from the commutator structure of the group. All of this paper is devoted to their proofs. The two equations in (iii) arise from the power structure. They are quite easy to prove but they are troublesome when one tries to draw up a list of all the groups for they split into a variety of.cases:

Corollary. Suppose we take $a(k)=\bar{a}(k)=1$ so $\delta=\alpha^{k-2}$. Then:

(i) If $2 k-3<n-1$ then $\beta=0$ and the equations in (iii) reduce to the four cases:

$$
\begin{aligned}
& z=\bar{z}=0, \quad z=\bar{z}=0, \quad z=\alpha^{n-2} \bar{z}, \quad z=\alpha^{n-2} \bar{z}, \\
& w=\bar{w}=0, \quad w=\alpha^{n-k-5} \bar{w}, \quad w=\bar{w}=0, \quad w=\alpha^{n-k-5} \bar{w} .
\end{aligned}
$$

The parameters $z$ and $w$, except for the cited cases, are not zero.

(ii) If $2 k-3>n$ and $k>n-p+3$, there is a reduction to three cases:

$$
\begin{array}{lll}
z=\bar{z}=0, & z=\bar{z}=0, & z=\alpha^{n-2} \bar{z}, \\
w=\bar{w}=0, & w=\alpha^{n-k-5} \bar{w}, & w=\bar{w}=0, \\
\beta \text { arbitrary, } & \beta \text { arbitrary, } & \beta=0 .
\end{array}
$$

(iii) If $k=n-p+2$ there is a reduction to two cases:

$$
\begin{array}{ll}
z=\bar{z}=0, & z=\bar{z}=0, \\
w=\bar{w}=0, & w=\alpha^{n-k-5} \bar{w}, \\
\beta=0, & \beta=0 .
\end{array}
$$

The comment " $\beta$ is arbitrary" in the first two cases of part (ii) means that the isomorphism equations are independent of $\beta$. In the other cases normalization of a parameter forces one to take $\beta=0$.

The following result enables one to select the distinct groups in a class:

Theorem 3. Let $(j(1), \ldots, j(t))$ be a fixed set of positive integers. Let

$$
B(t)=\left\{\left(b_{1}, \ldots, b_{t}\right): 1<b_{i}<p-1, i=1, \ldots, t\right\} .
$$

Define an equivalence relation on $B(t)$ by $\left(a_{1}, \ldots, a_{t}\right) \sim\left(b_{1}, \ldots, b_{t}\right)$ if and only if there is an integer $\alpha$ such that

$$
a_{i} \equiv \alpha^{j(i)} b_{i} \bmod (p-1), \quad i=1, \ldots, t .
$$

Let $\lambda(i)=(j(1), \ldots, j(i-1), p-1)$ for $i>2$. Let $L=\left\{\left(l_{1}, \ldots, l_{t}\right)\right\}$ be the set of integral $t$-tuples defined by 


$$
\begin{aligned}
& 0<l_{1}<(p-1, j(1)), \\
& 0<l_{i}<\left(\frac{(p-1) j(i)}{\lambda(i)}, p-1\right), \quad i=2, \ldots, t .
\end{aligned}
$$

Let $g$ be a primitive root modulo $p$. Then $\left\{\left(g^{l_{1}}, \ldots, g^{l_{1}}\right):\left(l_{1}, \ldots, l_{t}\right) \in L\right\}$ is a complete set of distinct representatives of the equivalence classes of $B(t)$ relative to $(j(1), \ldots, j(t))$. In addition, there are exactly $(j(1), \ldots, j(t), p-1)(p-$ $1)^{t-1}$ such equivalence classes.

A list of our groups can be obtained from Theorem 2, the corollary, and Theorem 3. Suppose for example we take the second subcase of part (ii) of the corollary. That is, the power equations are $z=\bar{z}=0$ and $w=\alpha^{n-k-5} \bar{w}$. Then the isomorphism equations for this case are

$$
\begin{gathered}
a_{i}(q(i))=\alpha^{q(i)-q(i-1)} \bar{a}_{i}(q(i)), \quad i=1, \ldots, h, \\
w=\alpha^{n-k-5} \bar{w} .
\end{gathered}
$$

So, set $j(i)=q(i)-q(i-1)$ for $i=1, \ldots, h$ and let $j(h+1)=n-k-5$. Then the nonisomorphic groups are given by

$$
a_{i}(q(i))=g^{4}, \quad i=1, \ldots, h ; \quad w=g^{h+1} ; \quad z=0 .
$$

In addition, there are

$$
(q(1)-q(0), \ldots, q(h)-q(h-1), n-k-5, p-1)(p-1)^{h}
$$

such groups. Each corresponds to a particular selection of $\left(l_{1}, \ldots, l_{h+1}\right)$.

As mentioned, the groups of Theorem 1 can also be described in terms of ideals. Szekeres [5] employed an ideal approach for the construction of all two-generator metabelian p-groups. But, as he stated, his method left a "residual" isomorphism problem.

The first subcase of part (ii) of the corollary illustrates the different ideals in a simple case. We have $\bar{z}=\bar{w}=0$, the multiple sum in (1) reduces to a single sum, $a_{i}(q(i))=g^{l}$, and $P(l)=g^{l_{0}+\cdots+l}$ where $l_{0}=0$. Consequently

$$
(y-1)=\sum_{\nu=0}^{h} g^{l_{0}+\cdots+\zeta} \sum_{i=q(\nu)}^{n-1} \frac{(q(\nu)-2) !}{(i-2) !} S_{i-2}^{q(\nu)-2}(x-1)^{i-2} .
$$

In sum, given $q(0), \ldots, q(h)$ one has a sequence of polynomials in $x-1$ that depend only on $q(0), \ldots, q(h)$, the inner sums above. The ideals corresponding to the distinct groups are obtained by letting $\left(l_{1}, \ldots, l_{h}\right)$ vary over $L$.

The methods of this paper can be used to determine the automorphism group of the groups of Theorem 1. We shall see (Lemma 1.1) that, if $\bar{G}$ is isomorphic to $G$ under $\theta$ and $\tau$ is the map from $\bar{G}$ to $G$ defined by

$$
\bar{x}^{\tau}=\bar{x}^{\theta} g, \quad \bar{y}^{\tau}=\bar{y}^{\theta} h,
$$


where $g$ and $h$ are arbitrary fixed elements of $G_{2}$, then $\tau$ is also an isomorphism from $\bar{G}$ to $G$. Next, if we take $\bar{G}=G, \bar{x}=x$, and $\bar{y}=y$ in Theorem 1 , then $\bar{a}_{i}(q(i))=a_{i}(q(i))$. Thus the automorphism $\vartheta$, where

$$
\bar{x}^{\vartheta}=x^{\hat{q}} y^{\beta}, \bar{y}^{\vartheta}=y^{\delta},
$$

is determined by the equations

$$
\begin{gathered}
\delta=\alpha^{k-2}, \quad \alpha^{q(i)-q(i-1)}=1, \quad i=1, \ldots, h, \\
\varepsilon \alpha^{n-2}=\varepsilon, \quad \eta \alpha^{n-k-5}=\eta
\end{gathered}
$$

when $\varepsilon=0$ or 1 and $\eta=0$ or 1 , the particular values being determined by the subcases of the corollary to Theorem 2 . In addition, $\beta=0$ or $\beta$ is arbitrary depending, again, on the subcases.

Consequently, if $G$ is in $\mathcal{C}(q(0), \ldots, q(h))$ then

$$
\begin{aligned}
\mid \text { Aut } G \mid=(q(1)-q(0), & \ldots, q(h)-q(h-1), \\
& \varepsilon(n-2), \eta(n-k-5), p-1) p^{2 n-2+b},
\end{aligned}
$$

where $b=0$ if $\beta=0$ and $b=1$ when $\beta$ is arbitrary. These results have several easy consequences: $S_{p}$ the Sylow $p$-group of Aut $G$ is normal, for the number of $S_{p}$ is congruent to $1 \bmod p$ and divides $p-1$; the complement of $S_{p}$ in Aut $G$ is cyclic, for it is a subgroup of the reduced residues modulo $p$. Finally, in the cases where $\beta=0$ and Aut $G$ has a $p^{\prime}$ automorphism, i.e., the common divisor above is not 1 , the group $G$ has exactly two characteristic subgroups of index $p,\left\langle x, G_{2}\right\rangle$ and $\left\langle y, G_{2}\right\rangle$.

I am indebted to the referee for a number of suggestions which led to marked improvements in the proofs of this paper.

1. This section is on the normalization of isomorphisms. There is but one result:

LeMMA 1.1. Suppose $G=\langle x, y\rangle$ and $\bar{G}=\langle\bar{x}, \bar{y}\rangle$ are defined as in Theorem 1 and $\theta$ is an isomorphism from $G$ to $\bar{G}$. Let $\bar{g}$ and $\bar{h}$ be any fixed elements of $\bar{G}_{2}$. Define $a$ map $\psi$ at $x$ and at $y$ by

$$
x^{\psi}=x^{\theta} \bar{g}, \quad y^{\psi}=y^{\theta} \bar{h} .
$$

Extend $\psi$ to the basis elements of $G_{2}$ by setting

$$
u_{i}^{\psi}=\left[y^{\psi}, x^{\psi}, \ldots i-i, x^{\psi}\right]
$$

for $i>2$. Finally, extend $\psi$ to all of $G$ by setting

$$
\left(x^{b(0)} y^{b(1)} u_{2}^{b(2)} \cdots u_{n-1}^{b(n-1)}\right)^{\psi}=\left(x^{\psi}\right)^{b(0)}\left(y^{\psi}\right)^{b(1)}\left(u_{2}^{\psi}\right)^{b(2)} \cdots\left(u_{n-1}^{\psi}\right)^{b(n-1)} \text {, }
$$

for all integers $b(0), \ldots, b(n-1)$ with $0 \leqslant b(0), \ldots, b(n-1)<p-1$. Then $\psi$ is an isomorphism from $G$ to $\bar{G}$. 
The direction of the maps in Lemma 1.1 was selected for typographical reasons. The direction will be reversed in the applications.

This result appeared in [2]. We sketch a proof for the sake of completeness. The usual commutator relations $[a b, c]=[a, c]^{b}[b, c],[a, b c]=[a, c][a, b]^{c}$, and the fact that $G$ is metabelian will be used without further comment.

To start,

$$
u_{2}^{\psi}=\left[y^{\psi}, x^{\psi}\right]=\left[y^{\theta} \bar{h}, x^{\theta} \bar{g}\right]=u_{2}^{\theta} a^{\theta},
$$

where $a=[y, g][h, x], g^{\theta}=\bar{g}$, and $h^{\theta}=\bar{h}$. Thus, by induction,

$$
u_{i}^{\psi}=u_{i}^{\theta}[a, x, \ldots, i, x]^{\theta} \text { for } i>2 .
$$

For $i=n-1$ we have, since $a \in G_{3}, u_{n-1}^{\psi}=u_{n-1}^{\theta}$. Since $G_{n-1}$ is of exponent $p$ it follows that $\psi$ is an isomorphism on $G_{n-1}$.

It is quite easy to prove that if $\psi$ is an isomorphism on $G_{i+1}$, where $i>2$, then it is also an isomorphism on $G_{i}$. The isomorphism equations for the induction reduce to a trivial generalization of part (iv) of Theorem 1 .

The extension of $\psi$ to $G_{1}$ is based on

$$
\begin{gathered}
\left(y^{p}\right)^{\psi}=\left(y^{\psi}\right)^{p}, \\
{\left[u_{j}, y\right]^{\psi}=\left[u_{j}^{\psi}, y^{\psi}\right] \quad \text { for } j>2 .}
\end{gathered}
$$

The proof of (1) rests on the fact that $G_{k}$ is of exponent $p$ and $G_{k+p-2}=1$. If one starts with $\left(y^{p}\right)^{\psi}$, applies part (iii) of Theorem 1 , and uses the calculated values of $u_{i}^{\psi}$ one has

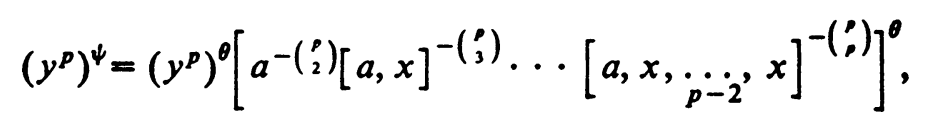

where $a=[y, g][h, x]$. Since $\left(y^{\psi}\right)^{p}=\left(y^{\theta} \bar{h}\right)^{p}=\left((y h)^{p}\right)^{\theta}$, it follows that $\left(y^{\psi}\right)^{p}$ $=\left(y^{p}\right)^{\psi}$ if and only if $y^{p} A B=(y h)^{p}$, where

$$
A=[y, g]^{-\left(\begin{array}{l}
p \\
2
\end{array}\right)}[y, g, x]^{-\left(\begin{array}{l}
( \\
3
\end{array}\right)} \ldots[y, g, x, \ldots, \ldots, x]^{-\left(\begin{array}{l}
0 \\
?
\end{array}\right)}
$$

and

$$
B=[h, x]^{-\left(\begin{array}{l}
p \\
2
\end{array}\right)}[h, x, x]^{-\left(\begin{array}{l}
p \\
3
\end{array}\right)} \ldots[h, x, \ldots, \ldots, x]^{-\left(\begin{array}{l}
p \\
p
\end{array}\right)} \text {. }
$$

To continue, $A=1$ for $[y, g] \in G_{k}, G_{k}$ is of exponent $p$, and $G_{k+p-2}=1$. Next, by part (iv) of Theorem $1, B=h^{p}$. Thus the problem reduces to showing that $(y h)^{p}=y^{p} h^{p}$. But this is true, for $(y h)^{p}=y^{p} h^{\tau}$, where

$$
\begin{aligned}
\tau & =1+y+\cdots+y^{p-1} \\
& =\left(\begin{array}{l}
p \\
1
\end{array}\right)+\left(\begin{array}{l}
p \\
2
\end{array}\right)(y-1)+\cdots+\left(\begin{array}{l}
p \\
p
\end{array}\right)(y-1)^{p-1} .
\end{aligned}
$$

Verification of (2) is based on the relation 


$$
y-1=\sum_{i>k} a(i)(x-1)^{i-2}
$$

and the computed values of $u_{i}^{\psi}$.

The extension of $\psi$ from $G_{1}$ to $G$ is similar. For example, to prove that $\left(x^{p}\right)^{\psi}=\left(x^{\psi}\right)^{p}$ one starts from part (iii) of Theorem 1: $\left(x^{p}\right)^{\psi}=\left(u_{n-1}^{w}\right)^{\psi}=$ $\left(u_{n-1}^{\theta}\right)^{w}$. Then, by Lemma 13-d of [3, p. 368], $(x g)^{p}=x^{p}$ so $\left(x^{\psi}\right)^{p}=\left(x^{\theta} g^{\theta}\right)^{p}$ $=\left(x^{p}\right)^{\theta}=\left(u_{n-1}^{N}\right)^{\theta}$.

This completes the sketch of the proof of Lemma 1.

Applying it, and using the fact that $G_{1}=\left\langle y, G_{2}\right\rangle$ is a characteristic subgroup of $G$, we have the

COROLLARY. Suppose $G$ and $\bar{G}$ are isomorphic. Then there is an isomorphism $\checkmark$ from $\bar{G}$ to $G$ that is of the form

$$
\bar{x}^{\ominus}=x^{\alpha} y^{\beta}, \bar{y}^{\vartheta}=y^{\delta} .
$$

2. This is a reference section on Stirling numbers. Starting from the definitions

$$
(x)_{n}=\sum_{m=0}^{n} S_{n}^{m} x^{m}, \quad x^{n}=\sum_{m=0}^{n} \delta_{n}^{m}(x)_{m}
$$

we have the well-known, easily proved identities:

$$
\begin{aligned}
& \sum_{m=i}^{j} S_{m}^{i} \delta_{j}^{m}=\delta_{i j}, \\
& \sum_{m=i}^{j} \delta_{m}^{i} S_{j}^{m}=\delta_{i j},
\end{aligned}
$$

where $\delta_{i}=1$ if $i=j$ and is 0 if $i \neq j$;

$$
\begin{aligned}
& S_{n+1}^{m}=S_{n}^{m-1}-n S_{n}^{m}, \\
& S_{n+1}^{m}=S_{n}^{m-1}+m \delta_{n}^{m} ;
\end{aligned}
$$

and for $m>n>1$,

$$
\begin{gathered}
(-1)^{m-n} \frac{m !}{n !} S_{n}^{m}=\sum_{\left(x_{1}, \ldots, x_{m}\right)} \frac{1}{x_{1} \cdots x_{m}}, \\
\frac{m !}{n !} \delta_{n}^{m}=\sum_{\left(x_{1}, \ldots, x_{m}\right)} \frac{1}{x_{1} ! \cdots x_{m} !},
\end{gathered}
$$

where the summation is on those integral $m$-tuples $\left(x_{1}, \ldots, x_{m}\right)$ with $x_{i}>1$ and $x_{1}+\cdots+x_{m}=n$.

To continue, we have

$$
\sum_{i=1}^{m}(-1)^{m-i}\left(\begin{array}{c}
m \\
i
\end{array}\right) i^{n}=m ! \delta_{n}^{m}
$$


To prove this expand $i^{n}$ in terms of Stirling numbers of the second type. Next,

$$
\begin{aligned}
& \sum_{t=i-j}^{n-m} \frac{m !}{(n-t) !} S_{n-t}^{m} \frac{i !}{(j+t) !} S_{j+t}^{i}=\frac{(i+m) !}{(j+n) !} S_{n+j}^{i+m}, \\
& \sum_{t=i-j}^{n-m} \frac{m !}{(n-t) !} S_{n-t}^{m} \frac{i !}{(j+t) !} S_{j+t}^{i}=\frac{(i+m) !}{(j+n) !} S_{n+j}^{i+m} .
\end{aligned}
$$

These follow directly from (5) and (6).

We shall need the following generalization of (8): Let $\sigma(m, i)=(m ! / i !) S_{i}^{m}$. Suppose $m_{i}>0$ and $m_{1}+\cdots+m_{n}=M$. Let $*$ denote summation over all nonnegative $i_{1}, \ldots, i_{n}$ with $i_{1}+\cdots+i_{n}=N$. Then

$$
\sum^{*} \sigma\left(m_{1}, i_{1}\right) \cdots \sigma\left(m_{n}, i_{n}\right)=\sigma(M, N) .
$$

LEMMA 2.1. If $N>j+c$ then

$$
\sum_{l=j+c}^{N}(l)_{c} S_{l-c}^{j} \delta_{N}^{l}=\left(\begin{array}{l}
N \\
j
\end{array}\right) c ! \delta_{N-j}^{c}
$$

and

$$
\sum_{l=j+c}^{N}(l)_{c} \mathcal{S}_{l-c}^{j} S_{N}^{l}=\left(\begin{array}{c}
N \\
j
\end{array}\right) c ! S_{N-j}^{c} \text {. }
$$

The first equation here is Lemma 2.3 of [3]. The proof there is complicated. The following simple proof is due to C. L. Chang: Write (9) as

$$
\left(\begin{array}{l}
l \\
c
\end{array}\right) \delta_{N}^{\prime}=\sum_{i=l-c}^{N-c}\left(\begin{array}{c}
N \\
i
\end{array}\right) \delta_{N-i}^{c} \delta_{i}^{l-c} .
$$

Then multiply this equation by $S_{l-c}^{j}$, sum from $l=j+c$ to $l=N$, reverse the order of summation of the double sum, and apply (2). This does it.

The proof of the second equation of Lemma 2.1 is similar.

LEMMA 2.2. If $a+b=t$ and $a \geqslant 1$ then

$$
\begin{aligned}
\sum_{\omega=t-1}^{i} \frac{t !}{(\omega+1) !} & S_{\omega+1}^{t} \frac{\omega !}{i !} \delta_{i}^{\omega} \\
& =\sum_{\omega=a-1}^{i-b} \frac{a !}{(\omega+1) !} S_{\omega+1}^{a} \frac{\omega !}{(i-b) !} \delta_{i-b}^{\omega} .
\end{aligned}
$$

Proof. To start, by (8),

$$
\frac{t !}{(\omega+1) !} S_{\omega+1}^{t}=\sum_{j=a}^{\omega+1-b} \frac{a !}{j !} S_{j}^{a} \frac{b !}{(\omega+1-j) !} S_{\omega+1-j}^{b},
$$

where $a$ and $b$ are any nonnegative integers with $a+b=t$. To finish 
multiply the above equation by $(\omega ! / i !) \S_{i}^{\omega}$, add, and apply Lemma 2.1.

LEMMA 2.3. We have

$$
\sum_{p=r}^{z}\left(\begin{array}{l}
z \\
p
\end{array}\right) \mathcal{S}_{p}^{r}=\mathcal{S}_{z+1}^{r+1}
$$

and

$$
\sum_{p=r}^{z-1}\left(\begin{array}{l}
z \\
p
\end{array}\right) \mathcal{S}_{p}^{r}=(r+1) \mathcal{S}_{z}^{r+1}
$$

Proof. To start,

$$
\left(\begin{array}{l}
z \\
p
\end{array}\right) x^{p}=\sum_{r=0}^{p}\left(\begin{array}{l}
z \\
p
\end{array}\right) \mathfrak{S}_{p}^{r}(x)
$$

Thus

$$
(1+x)^{z}=\sum_{p=0}^{z}\left(\begin{array}{l}
z \\
p
\end{array}\right) x^{p}=\sum_{r=0}^{z}(x)_{r} \sum_{p=r}^{z}\left(\begin{array}{l}
z \\
p
\end{array}\right) \delta_{p}^{r}
$$

Next,

$$
(1+x)^{2}=\sum_{r=0}^{z} \mathcal{S}_{2}^{r}(1+x)_{r}=\sum_{r=0}^{z}\left(\mathcal{S}_{z}^{r}+(r+1) \mathcal{S}_{z}^{r+1}\right)(x)_{r}
$$

So, equating coefficients and then using (4) we have

$$
\sum_{p=r}^{z}\left(\begin{array}{l}
z \\
p
\end{array}\right) \delta_{p}^{r}=\delta_{z}^{r}+(r+1) \delta_{z}^{r+1}=\delta_{z+1}^{r+1} \text {. }
$$

This proves the first equation. The second follows from the first.

LEMMA 2.4. Set

$$
f^{*}(r, \nu, \omega)=\sum_{m=0}^{r}(-1)^{r-m}\left(\begin{array}{c}
r \\
m
\end{array}\right)\left(\begin{array}{c}
i+m a \\
\nu
\end{array}\right)\left(\begin{array}{c}
j+m b \\
\omega
\end{array}\right) .
$$

Let

$$
\gamma(\nu, x, i)=\sum_{e=x}^{\nu}(\nu-e) \frac{S_{e}^{x}}{e !}
$$

Then

$$
f^{*}(r, \nu, \omega)=r ! \sum_{x=0}^{\nu} \sum_{y=0}^{\omega} a^{x} b^{y} \mathcal{f}_{x+y}^{r} \gamma(\nu, x, i) \gamma(\omega, y, j)
$$

Proof. If we use Vandermonde's convolution and then reverse the order of summation we have

$$
f^{*}(r, \nu, \omega)=\sum_{e=0}^{\nu} \sum_{f=0}^{\omega}\left(\begin{array}{c}
i \\
\nu-e
\end{array}\right)\left(\begin{array}{c}
j \\
\omega-f
\end{array}\right) \pi(r)
$$


where

$$
\pi(r)=\sum_{m=0}^{r}(-1)^{r-m}\left(\begin{array}{c}
r \\
m
\end{array}\right)\left(\begin{array}{c}
m a \\
e
\end{array}\right)\left(\begin{array}{c}
m b \\
f
\end{array}\right) .
$$

Expanding by Stirling numbers of the first kind, reversing the order of summation, and applying (7), we find that

$$
\begin{aligned}
\pi(r) & =\sum_{x=0}^{e} \sum_{y=0}^{f} \frac{S_{e}^{x}}{e !} \frac{S_{f}^{y}}{f !} a^{x} b^{y} \sum_{m=0}^{r}(-1)^{r-m}\left(\begin{array}{c}
r \\
m
\end{array}\right) m^{x+y} \\
& =\sum_{x=0}^{e} \sum_{y=0}^{f} \frac{S_{e}^{x}}{e !} \frac{S_{f}^{y}}{f !} a^{x} b^{y} r ! S_{x+y}^{r} .
\end{aligned}
$$

Combining these results we get the conclusion of Lemma 3.6.

COROllary. If $\nu+\omega<r$ then $f^{*}(r, \nu, \omega)=0$.

Proof. If $\nu+\omega<r$ then $x+y<\nu+\omega<r$, so $\mathfrak{S}_{x+y}^{r}=0$.

LEMMA 2.5. Let

$$
f(r, \nu, \omega)=\sum_{m=0}^{r} \sum_{i=0}^{\alpha-1} \sum_{j=\beta}^{\delta+\beta-1}(-1)^{r-m}\left(\begin{array}{c}
r \\
m
\end{array}\right)\left(\begin{array}{c}
i+m a \\
\nu
\end{array}\right)\left(\begin{array}{c}
j+m b \\
\omega
\end{array}\right) .
$$

Set

$$
\theta(u, v ; \nu, x)=\sum_{q=1}^{v+1-x}\left(\begin{array}{c}
x+q \\
q
\end{array}\right) \frac{S_{\nu+1}^{x+1}}{(\nu+1) !}\left[(u+v)^{q}-v^{q}\right]
$$

Then

$$
f(r, \nu, \omega)=\sum_{x=0}^{\nu} \sum_{y=0}^{\omega} r ! a^{x} b^{y} \delta_{x+y}^{r} \theta(\alpha, 0 ; \nu, x) \theta(\delta, \beta ; \omega, y) .
$$

Proof. Since $f(r, \nu, \omega)$ is a sum of the $f^{*}(r, \nu, \omega)$ we have, by Lemma 2.4,

$$
f(r, \nu, \omega)=\sum_{x=0}^{\nu} \sum_{y=0}^{\omega} r ! a^{x} b^{y} \mathcal{S}_{x+y}^{r} A B,
$$

where

$$
A=\sum_{e=x}^{\nu} \frac{S_{e}^{x}}{e !} \sum_{i=0}^{\alpha-1}(\nu-e)=\sum_{e=x}^{\nu} \frac{S_{e}^{x}}{e !}\left(\begin{array}{c}
\alpha \\
\nu+1-e
\end{array}\right)
$$

and

$$
\begin{aligned}
B & =\sum_{f=y}^{\omega} \frac{S_{f}^{y}}{f !} \sum_{j=\beta}^{\delta+\beta-1}\left(\begin{array}{c}
j \\
\omega-f
\end{array}\right) \\
& =\sum_{f=y}^{\omega} \frac{S_{f}^{y}}{f !}\left[\left(\begin{array}{c}
\delta+\beta \\
\omega+1-f
\end{array}\right)-\left(\begin{array}{c}
\beta \\
\omega+1-f
\end{array}\right)\right] .
\end{aligned}
$$


As for $A$, expanding by Stirling numbers of the first kind and rearranging we have

$$
A=\sum_{q=1}^{\nu+1-x} \alpha^{q} \sum_{e=x}^{\nu+1-q} \frac{S_{e}^{x}}{e !} \frac{S_{\nu+1-e}^{q}}{(\nu+1-e) !}
$$

Thus by (8)

$$
A=\sum_{q=1}^{\nu+1-x} \alpha^{q} \frac{(x+q) !}{x ! q !} \frac{S_{\nu+1}^{x+q}}{(\nu+1) !}=\theta(\alpha, 0 ; \nu, x) .
$$

Similarly, $B=\theta(\delta, \beta ; \omega, y)$.

3. In this section we shall derive the isomorphism equations that come out of the commutator structure of the group.

LEMMA 3.1. Suppose that $\bar{G}=\langle\bar{x}, \bar{y}\rangle$ and $G=\langle x, y\rangle$ are isomorphic under $\vartheta$, where $\bar{x}^{\vartheta}=x^{\mathcal{\theta}} y^{\beta}$ and $\bar{y}^{\vartheta}=y^{\delta}$. Suppose that

$$
\bar{y}-1=\sum_{i>k} \bar{a}(i)(\bar{x}-1)^{i-2} \text {. }
$$

Set $\tau(u, m)=1+u+\cdots+u^{m-1}$ and $H(x, y)=\tau(x, \alpha) \tau(y, \delta) y^{\beta}$. Then, considering $x-1$ and $y-1$ as operators on $G_{2}$,

$$
H(x, y)\left(y^{\delta}-1\right)=H(x, y) \sum_{i>k} \bar{a}(i)\left(x^{\alpha} y^{\beta}-1\right)^{i-2} .
$$

Proof. We have

$$
\bar{u}_{2}^{*}=\left[\bar{y}^{\vartheta}, \bar{x}^{\vartheta}\right]=\left[y^{\delta}, x^{\alpha} y^{\beta}\right]=\left[y^{\delta}, x^{\alpha}\right]^{y^{\beta}} .
$$

In addition,

$$
\begin{gathered}
{\left[y^{\delta}, x^{\alpha}\right]=y^{-\delta}\left(y^{x^{\alpha}}\right)^{\delta}, \quad y^{x^{\alpha}}=y u_{2}^{\tau(x, \alpha)},} \\
\left(y^{x^{*}}\right)^{\delta}=y^{\delta} u_{2}^{\tau(x, \alpha) \tau(y, \delta)} .
\end{gathered}
$$

Thus $\bar{u}_{2}^{\vartheta}=u_{2}^{H(x, y)}$. To complete the proof apply $\vartheta$ to the equation

$$
\left[\bar{u}_{2}, \bar{y}\right]=\prod_{i>k}\left[\bar{u}_{2}, \bar{x}, \ldots, \dot{i}, \bar{x}\right]^{\bar{a}(i)} \text {. }
$$

The following result will be needed to determine the power structure equations.

LEMMA 3.2. Let $c=\alpha^{n-2} \delta$. Then $\bar{u}_{n-1}^{\theta}=u_{n-1}^{c}$.

Proof. According to the proof of Lemma 3.1, $\bar{u}_{2}^{\theta}=u_{2}^{H(x y)}$. Consequently,

$$
\bar{u}_{n-1}^{\vartheta}=\left[\bar{u}_{2}^{\vartheta}, \bar{x}^{\bullet}, \ldots, \bar{x}^{\bullet}\right]=u_{2}^{d},
$$

where $d=H(x, y)\left(x^{\alpha} y^{\beta}-1\right)^{n-3}$. To complete the proof expand $d$ in powers of $x-1$ and $y-1$. 
We now turn to the main problem at hand, rewriting (1) in powers of $x-1$ and $y-1$.

Lemma 3.3. Let $A(0,0)=1$ and $A(0, n)=0$ for $n>1$. Define $A(m, n)$ for $m>1$ by

$$
A(m, n)=\sum_{i_{1}, \ldots, i_{m}}^{*} a\left(i_{1}\right) \cdots a\left(i_{m}\right),
$$

where $*$ indicates the summation is over those $i_{1}, \ldots, i_{n}>k$ with $i_{1}+\cdots+$ $i_{m}=n+2 m$. Then for $m \geqslant 0$,

$$
(y-1)^{m}=\sum_{n>m(k-2)} A(m, n)(x-1)^{n} .
$$

The proof is obvious.

Several functions need to be defined. Set

$$
g(1, \nu, \omega)
$$

$$
=\sum_{z=1}^{\nu+1} \sum_{r=2}^{\omega+1} \sum_{s=0}^{\omega+1-r} \alpha^{z} \delta^{r} \beta^{s} \frac{S_{\nu+1}^{z}}{(\nu+1) !} \frac{S_{\omega+1}^{r+s}}{(\omega+1) !}\left(\begin{array}{c}
r+s \\
r
\end{array}\right) 2 ! \delta_{r}^{2} .
$$

Let, for $r \geq 2$,

$$
\zeta_{r}(z, u, v)=\sum_{q=0}^{z-1} \sum_{t=u}^{u+v}\left(\begin{array}{l}
z \\
q
\end{array}\right)\left(\begin{array}{c}
u+v \\
t
\end{array}\right)\left(\begin{array}{l}
t \\
u
\end{array}\right) r ! \delta_{v+u+q-t}^{r}
$$

and

$$
\begin{aligned}
& g(r, \nu, \omega) \\
& \quad=\sum_{z=1}^{\nu+1} \sum_{u=1}^{\omega+1} \sum_{v=0}^{\omega+1-u} \alpha^{z} \delta^{u} \beta^{v} \frac{S_{\nu+1}^{z}}{(\nu+1) !} \frac{S_{\omega+1}^{u+v}}{(\omega+1) !} \zeta_{r}(z, u, v) .
\end{aligned}
$$

We can now state the main result of this section. It will be derived by writing (1) in powers of $(x-1)$ and $(y-1)$ and then comparing coefficients.

LeMma 3.4. Let $g(1, \nu, \omega), g(i, \nu, \omega)$, and $A(m, n)$ be defined as above. Given $N>k-2$, let $N^{\prime}=[N /(k-2)]$. Set

$$
\begin{aligned}
L(N) & =\sum_{\omega=1}^{N^{\prime}} \sum_{n=\omega(k-2)}^{N} A(\omega, n) g(1, N-n, \omega), \\
R_{1}(N) & =\sum_{i=k}^{N+2} \bar{a}(i) g(i-2, N, 0),
\end{aligned}
$$

and

$$
R_{2}(N)=\sum_{i=k}^{N+5-k} \bar{a}(i) \sum_{\omega=1}^{N^{\prime}} \sum_{n=\omega(k-2)}^{N} A(\omega, n) g(i-2, N-n, \omega) .
$$


Then (1) is equivalent to the set of equations

$$
L(N)=R_{1}(N)+R_{2}(N), \quad N=k-2, \ldots, n-3 .
$$

LEMMA 3.5. Set

$$
h(r)=\tau(x, \alpha) \tau(y, \delta) y^{\beta}\left(x^{a} y^{b}-1\right)^{r} .
$$

Let

$$
f(r, \nu, \omega)=\sum_{m=0}^{r} \sum_{i=0}^{\alpha-1} \sum_{j=\beta}^{\delta+\beta-1}(-1)^{r-m}\left(\begin{array}{l}
r \\
m
\end{array}\right)\left(\begin{array}{c}
i+m a \\
\nu
\end{array}\right)\left(\begin{array}{c}
j+m b \\
\omega
\end{array}\right) .
$$

Let

$$
S_{1}=\sum_{N>r}(x-1)^{N} f(r, N, 0)
$$

and

$$
S_{2}=\sum_{N>r+k-3}(x-1)^{N} \sum_{\omega=1}^{N^{\prime}} \sum_{n=\omega(k-2)}^{N} A(\omega, n) f(r, N-n, \omega),
$$

where $N^{\prime}=[N /(k-2)]$. Then $h(r)=S_{1}+S_{2}$.

Proor. First express $h(r)$ as a polynomial in $(x-1)$ and $(y-1)$ :

$$
h(r)=\sum_{\nu>0} \sum_{\omega>0} f(r, \nu, \omega)(x-1)^{\nu}(y-1)^{\omega}
$$

where $f(r, \nu, \omega)=0$ for $\nu+\omega<r$. Second, split off those terms where $\omega=0$ to form $S_{1}$. Finally, apply Lemma 3.3 to those terms where $\omega>1$; this yields $\boldsymbol{S}_{\mathbf{2}}$.

LemaA 3.6. Let $L(N)$ be defined in Lemma 3.4. Then

$$
H(x, y)\left(y^{8}-1\right)=\sum_{N>k-2}(x-1)^{N} L(N) .
$$

Proof. Take $r=1, a=0$, and $b=\delta$ in Lemma 3.5. Let $g(1, \nu, \omega)$ be the corresponding value of $f(1, \nu, \omega)$. By Lemma 2.5, this value is the one given in (2). Next, by (2), $g(1, \nu, 0)=0$; thus the $S_{1}$ sum of Lemma 3.5 is zero. The $S_{2}$ sum corresponds to the one above.

LeMaM 3.7. Let $R_{1}(N)$ and $R_{2}(N)$ be defined as in Lemma 3.4. Then

$$
\begin{aligned}
\sum_{i>k} \bar{a}(i) H & (x, y)\left(x^{\alpha} y^{\beta}-1\right)^{i-2} \\
= & \sum_{N>k-2}(x-1)^{N} R_{1}(N)+\sum_{N>2 k-5}(x-1)^{N} R_{2}(N) .
\end{aligned}
$$

Proof. First, take $a=\alpha, b=\beta$ and let $g(r, \nu, \omega)$ be the resulting value of $f(r, v, \omega)$. Then the two sums of Lemma 3.5 correspond to the two sums 
above. The fact that $f(r, \nu, \omega)$ is the $g(r, \nu, \omega)$ given in (3) follows from Lemma 2.5.

Lemma 3.4 follows from Lemmas 3.6 and 3.7.

The first block of equations in Lemma 3.4 takes the following form:

LEMMA 3.8. Let $\varepsilon(N)=0$ for $N=k-2, \ldots, 2 k-6$ and $\varepsilon(2 k-5)=(k$ - 2) $a(k) \bar{a}(k) \beta$. Then the equations of Lemma 3.4 corresponding to $N=k-$ $2, \ldots, 2 k-5$ are equivalent to the set $\delta a(k)=\alpha^{k-2} \bar{a}(k)$ and, for $N=k-$ $2, \ldots, 2 k-5$,

$$
\begin{aligned}
\sum_{q=1}^{N+3-k} \alpha^{q} \sum_{i=k}^{N+3-q} \frac{a(i)}{a(k)} \frac{S_{N+3-i}^{q}}{(N+3-i) !} \\
=\sum_{q=1}^{N+3-k} \alpha^{q} \sum_{i=k}^{k+q-1} \frac{\bar{a}(i)}{\bar{a}(k)} \frac{S_{N+1}^{k+q-2}}{(N+1) !}(i-1) ! \delta_{k+q-2}^{i-1}+\frac{\varepsilon(N)}{\bar{a}(k)} .
\end{aligned}
$$

The equation corresponding to $N=k-2$ above is vacuous; it is included for ease in indexing later. There are several steps to the proof of Lemma 3.8. The first is

Lemai 3.9. If $N<2 k-5$ then

$$
L(N)=\delta^{2} \sum_{q=1}^{N+3-k} \alpha^{q} \sum_{i=k}^{N+3-q} a(i) \frac{S_{N+3-i}^{q}}{(N+3-i) !} .
$$

Proor. If $N<2 k-5$ then $N^{\prime}=[N /(k-2)]=1$ and

$$
L(N)=\sum_{n=k-2}^{N} A(1, n) g(1, N-n, 1) .
$$

Furthermore, $A(1, n)=a(n+2)$ and

$$
g(1, N-n, 1)=\delta^{2} \sum_{q=1}^{N-n+1} \alpha^{q} \frac{S_{N-n+1}^{q}}{(N-n+1) !} .
$$

Combining these results, we get Lemma 3.9.

LEMMA 3.10. For $N>k-2$,

$$
R_{1}(N)=\delta \sum_{z=k-1}^{N+1} \alpha^{z} \sum_{i=k}^{z+1} \bar{a}(i) \frac{S_{N+1}^{z}}{(N+1) !}(i-1) ! \delta_{z}^{i-1} .
$$

Proof. This follows from the fact that

$$
g(i-2, N, 0)=\delta \sum_{z=1}^{N+1} \alpha^{z} \frac{S_{N+1}^{z}}{(N+1) !} \zeta_{i-2}(z, 1,0)
$$

and, by Lemma 2.3 ,

$$
\zeta_{i-2}(z, 1,0)=\sum_{q=0}^{z-1}\left(\begin{array}{l}
z \\
q
\end{array}\right)(i-2) ! \S_{q}^{i-2}=(i-1) ! \delta_{z}^{i-1} .
$$


Leman 3.11. Let $R_{2}(N)$ be defined as in Lemma 3.4. Then

$$
R_{2}(2 k-5)=(k-2) \alpha^{k-2} \delta \beta a(k) \bar{a}(k) .
$$

Proof. By definition, $R_{2}(2 k-5)=\bar{a}(k) a(k) g(k-2, k-3,1)$. Then

$$
\begin{aligned}
g(k-2, k-3,1) & \\
& =\sum_{z=1}^{k-2} \sum_{u=1}^{2} \sum_{v=0}^{2-u} \alpha^{z} \delta^{u} \beta^{v} \frac{S_{k-2}^{2}}{(k-2) !} \frac{S_{2}^{u+v}}{2 !} \zeta_{k-2}(z, u, v),
\end{aligned}
$$

where

$$
\zeta_{k-2}(z, u, v)=\sum_{q=0}^{z-1} \sum_{t=u}^{u+v}\left(\begin{array}{l}
z \\
q
\end{array}\right)\left(\begin{array}{c}
u+v \\
t
\end{array}\right)\left(\begin{array}{l}
t \\
u
\end{array}\right)(k-2) ! \delta_{v+u+q-t}^{k-2}
$$

Next, from the indices of summation, $k-2>z, z>q+1$, and $v+u+q$ $-t>k-2$. Thus $u+v-t>1$. But then $u=v=t=1$. Consequently,

$$
g(k-2, k-3,1)=\sum_{z=1}^{k-2} \alpha^{z} \delta \beta \frac{S_{k-2}^{z}}{(k-2) !} \frac{1}{2 !} \zeta_{k-2}(z, 1,1),
$$

where

$$
\zeta_{k-2}(z, 1,1)=\sum_{q=0}^{z-1}\left(\begin{array}{l}
z \\
q
\end{array}\right)\left(\begin{array}{l}
2 \\
1
\end{array}\right)(k-2) ! \S_{q+1}^{k-2} .
$$

Lemma 3.11 follows from these results.

Lemma 3.8 follows from this sequence.

4. This section deals with the analysis of the isomorphism equations. Several functions need to be defined. Given two sequences $\{b(i)\}$ and $\{\bar{b}(i)\}$ define $F$ and $G$ by

$$
F(b, n, m)=\sum_{t=1}^{m+1-n} \alpha^{t} \sum_{i=n}^{m+1-t} b(i) \frac{S_{m+1-i}^{t}}{(m+1-i) !},
$$

(2) $\quad G(\bar{b}, n, m)=\sum_{t=1}^{m+1-n} \alpha^{i} \sum_{i=n}^{n+t-1} \bar{b}(i) \frac{S_{m-1}^{t+n-2}}{(m-1) !}(i-1) ! S_{t+n-2}^{i-1}$.

In addition, for typographical reasons, let

$$
\sigma(i, m)=(i ! / m !) S_{m}^{i}
$$

Leman 4.1. Let $F$ and $G$ be defined as above. Let $d(1)$ and $d(2)$ be nonzero parameters that do not depend on $m$. Suppose there is a nonzero $\alpha$ such that

$$
d(1) F(b, j, m)=d(2) G(\bar{b}, j, m), \quad m=j, \ldots, M .
$$

Suppose that $b(j)=\cdots=b(n-1)=0$. Then $\bar{b}(j)=\cdots=\bar{b}(n-1)=0$ and 


$$
d(1) b(n)=\alpha^{n-j} d(2) \bar{b}(n) .
$$

Thus $b(n)=0$ if and only if $\bar{b}(n)=0$. In addition, the equations in (3) corresponding to $m=j, \ldots, n-1$ are vacuous. Those corresponding to $m=$ $n, \ldots, M$ take the form

$$
d(1) F(b, n, m)=\alpha^{n-j} d(2) G(\bar{b}, n, m) .
$$

The proof of Lemma 4.1 is trivial; one need only reindex the sums in (1) and (2).

The analysis of our equations is based on

LEMMA 4.2. Let $n$ be a positive integer. Let $\{b(i): i>n\}$ and $\{\bar{b}(i): i>n\}$ be sequences with $b(n) \neq 0$ and $\bar{b}(n) \neq 0$. Suppose there is a nonzero $\alpha$ such that for $m=n, \ldots, M$,

$$
\frac{F(b, n, m)}{b(n)}+\frac{A(m)}{b(n)}=\frac{G(\bar{b}, n, m)}{\bar{b}(n)}+\frac{B(m)}{\bar{b}(n)},
$$

where $A(m)=B(m)=0$ for $m=n, \ldots, L$. Then there is an integer $\lambda, a$ sequence of integers $q(0), \ldots, q(\lambda)$ with $n=q(0)<\cdots<q(\lambda)<L$, and $a$ set of nonzero numbers $\left\{b_{i}(q(i)), \bar{b}_{i}(q(i))\right\}$ such that:

(i) The set of equations in (4) corresponding to $m=n, \ldots, L$ is equivalent to the system

$$
b_{i}(q(i))=\alpha^{q(i)-q(i-1)} \bar{b}_{i}(q(i)), \quad i=1, \ldots, \lambda .
$$

(ii) If $b_{0}(q(0))=b(n)$ and $P(\omega)=b_{0}(q(0)) \cdots b_{\omega}(q(\omega))$ then

$$
b(i)=\sum_{\omega=0}^{\lambda} P(\omega) \sigma(q(\omega)-2, i-2)
$$

for $i=n, \ldots, L$. Similarly, for $i=n, \ldots, L$,

$$
\bar{b}(i)=\sum_{\omega=0}^{\lambda} \bar{P}(\omega) \sigma(q(\omega)-2, i-2),
$$

where $\bar{P}(\omega)=\bar{b}_{0}(q(0)) \cdots \bar{b}_{\omega}(q(\omega))$ and $\bar{b}_{0}(q(0))=\bar{b}(n)$.

(iii) There are sequences $\{c(i)\}$ and $\{\bar{c}(i)\}$ with $c(i)=\bar{c}(i)=0$ for $i<L$ such that the equations of (4) corresponding to $m=L+1, \ldots, M$ reduce to

$$
\begin{aligned}
F(c, L+1, m) & =A(m) / P(\lambda) \\
& =\alpha^{L+1-q(\lambda)} G(\bar{c}, L+1, m)+\alpha^{n-q(\lambda)} B(m) / \bar{P}(\lambda) .
\end{aligned}
$$

Furthermore, for $i=L+1, \ldots, M$,

$$
b(i)=\sum_{\omega=0}^{\lambda} P(\omega) \sigma(q(\omega)-2, i-2)+P(\lambda) c(i)
$$

and 


$$
\bar{b}(i)=\sum_{\omega=0}^{\lambda} \bar{P}(\omega) \sigma(q(\omega)-2, i-2)+\bar{P}(\lambda) \bar{c}(i) .
$$

Proof. Define $b_{1}(i)$ for $i \geqslant n$ by

$$
b(i) / b(n)=\sigma(n-2, i-2)+b_{1}(i) .
$$

Define $\overline{b_{1}}(i)$ by analogy, i.e., replace each $b$ above by $\bar{b}$. Note, for later reference: $b_{1}(n)=\bar{b}_{1}(n)=0$.

To continue, if we divide (1) by $b(n)$ and employ the above definition, then the coefficient of $\alpha^{t}$ is equal to

$$
\sum_{i=n}^{m+1-t}\left[\frac{(n-2) !}{(i-2)} S_{i-2}^{n-2}+b_{1}(i)\right] \frac{S_{m+1-i}^{t}}{(m+1-i) !} .
$$

Consequently, by 2.8 , equation ( 8 ) of $\$ 2$,

$$
\frac{F(b, n, m)}{b(n)}=\sum_{t=1}^{m+1-n} \alpha^{t} \frac{(n-2+t) !}{(m-1) !} \frac{S_{m-1}^{n-2+t}}{t !}+F\left(b_{1}, n, m\right) .
$$

Similarly, by (2) and Lemma 2.1,

$$
\frac{G(\bar{b}, n, m)}{\bar{b}(n)}=\sum_{t=1}^{m+1-n} \alpha^{\prime} \frac{(n-2+t) !}{(m-1) !} \frac{S_{m-1}^{n-2+t}}{t !}+G\left(\bar{b}_{1}, n, m\right) .
$$

Thus the equations of (4) reduce to

$$
F\left(b_{1}, n, m\right)+A(m) / b(n)=G\left(\bar{b}_{1}, n, m\right)+B(m) / \bar{b}(n) .
$$

To continue, we have $b_{1}(n)=0$. Let $b_{1}(q(1))$ be the first nonzero element of the sequence $\left\{b_{1}(i)\right\}$ and suppose that $q(1)<L$. Then, by Lemma 4.1, $\bar{b}_{1}(n)=\cdots=\bar{b}_{1}(q(1)-1)=0, \bar{b}_{1}(q(1)) \neq 0$, and the equations in (5) reduce to

$$
b_{1}(q(1))=\alpha^{q(1)-n} \bar{b}_{1}(q(1))
$$

and, for $m \geqslant q(1)$,

$$
F\left(b_{1}, q(1), m\right)+\frac{A(m)}{b(n)}=\alpha^{q(1)-n} G\left(\bar{b}_{1}, q(1), m\right)+\frac{B(m)}{\bar{b}(n)} .
$$

Dividing the last equations by the first we obtain a set that has the form given in (4). Thus we can proceed by induction.

After $\lambda$ inductive steps we have a sequence $q(0), \ldots, q(\lambda)$ with $n=q(0)<$ $q(1)<\cdots<q(\lambda)<L$ and numbers $b_{j}(i)$ defined by $b_{0}(i)=b(i)$,

$$
\frac{b_{j-1}(i)}{b_{j-1}(q(j-1))}=\sigma[q(j-1)-2, i-2]+b_{j}(i)
$$

for $j=1, \ldots, \lambda$ and $i>q(j-1)$. We also have the equations of part (i) of 
Lemma 4.2 and a remaining set of equations of the form

$$
\frac{F\left(b_{\lambda}, q(\lambda), m\right)}{b_{\lambda}(q(\lambda))}+\frac{A(m)}{P(\lambda)}=\frac{G\left(\bar{b}_{\lambda}, q(\lambda), m\right)}{\bar{b}_{\lambda}(q(\lambda))}+\frac{\alpha^{n-q(\lambda)} B(m)}{\bar{P}(\lambda)}
$$

for $m>q(\lambda)$.

To conclude the proof of Lemma 4.2 set

$$
b_{\lambda}(i) / b_{\lambda}(q(\lambda))=\sigma[q(\lambda)-2, i-2]+c(i),
$$

assume that $c(q(\lambda))=\cdots=c(L)=0$, and argue as above to obtain the equations of part (iii). The given formulas for the $b(i)$ are obtained from the defining relations for the $b_{j}(i)$.

LEMMA 4.3. There is a sequence $q(0), \ldots, q\left(\lambda_{1}\right)$ with $k=q(0)<\cdots<$ $q\left(\lambda_{1}\right)<2 k-4$, a set of nonzero numbers $\left\{a_{i}(q(i)), \bar{a}_{i}(q(i))\right\}$, and a pair of numbers $\{c(2 k-3), \bar{c}(2 k-3)\}$ such that the system of equations of Lemma 3.8 is equivalent to the system:

$$
\begin{aligned}
\delta a(k) & =a^{k-2} \bar{a}(k), \\
a_{i}(q(i)) & =\alpha^{q(i)-q(i-1)} \bar{a}_{i}(q(i)), \quad i=k, \ldots, \lambda_{1}, \\
c(2 k-3) & =\alpha^{2 k-3} \bar{c}(2 k-3)+\frac{\alpha^{k-q\left(\lambda_{1}\right)}}{\bar{P}\left(\lambda_{1}\right)}(k-2) a(k) \bar{a}(k) \beta .
\end{aligned}
$$

In addition,

$$
a(i)=\sum_{\omega=0}^{\lambda_{1}} P(\omega) \sigma(q(\omega)-2, i-2), \quad i=1, \ldots, 2 k-4,
$$

and

$$
a(2 k-3)=\sum_{\omega=0}^{\lambda_{1}} P(\omega) \sigma(q(\omega)-2,2 k-5)+P(\lambda) c(2 k-3),
$$

where $P(\omega)=a_{0}(q(0)) \cdots a_{\omega}(q(\omega))$ and $a_{0}(q(0))=a(k)$.

Proof. Apply Lemma 4.2 with $b(i)=a(i), n=k, m=N+2, L=2 k-$ 4, $M=2 k-3, A(m)=0$ for all $m, B(m)=0$ for $m<2 k-4$ and $B(2 k-$ 3) $=(k-2) a(k) \bar{a}(k) \beta$.

To continue, if $|G|=p^{n}$ and $2 k-2>n$ then the equation of Lemma 4.3 for $c(2 k-3)$ does not appear in the list, and we are done. Thus we shall assume that $2 k-3<n-1$ for the balance of this section.

The third equation of Lemma 4.3 implies that we may take $c(2 k-3)=$ $\bar{c}(2 k-3)=0$. For if we start with $\bar{G}$ then, since $k-2 \neq 0$ and $\beta$ is linear, we can find an isomorphic image $G$ with $c(2 k-3)=0$. That is, since one member of the equivalence class of isomorphic images of $\bar{G}$ has its $c(2 k-3)$ 
component equal to zero, we may restrict our attention to those groups with $c(2 k-3)=\bar{c}(2 k-3)=0$. Once we normalize this way then, by the third equation again, we must have $\beta=0$.

Since Lemma 4.3 may be applied with $\bar{G}=G, \bar{x}=x$, and $\bar{y}=y$, the $c(2 k-3)=0$ condition means that $\left\langle x, G_{2}\right\rangle$ is a characteristic subgroup of $G$. To put it another way: There is a unique subgroup $H=\left\langle x, G_{2}\right\rangle \neq G_{1}$ of index $p$ such that $c(2 k-3)=0$ in the above expansion of $[y, x, y]$. For suppose $\bar{x}$ is any element such that $G=\left\langle\bar{x}, G_{1}\right\rangle$,

and

$$
[y, \bar{x}, y]=\bar{u}_{k}^{\bar{a}(k)} \ldots \bar{u}_{2 k-3}^{\bar{a}(2 k-3)} \bmod G_{2 k-2}
$$

$$
\bar{a}(i)=\sum_{\nu=0}^{\lambda_{1}} \bar{P}(\nu) \sigma[q(\nu)-2, i-2]
$$

for $i=k, \ldots, 2 k-3$. That is, $\bar{c}(2 k-3)=0$. Then, since $G=\langle x, y\rangle=$ $\langle\bar{x}, y\rangle$, there is an automorphism $\vartheta$ from $G$ to $G$ with $\bar{x}^{\theta}=x^{\alpha} y^{\beta}$ and $y^{\delta}=y^{\delta}$. Since $c(2 k-3)=\bar{c}(2 k-3)=0$ we have, by Lemma $4.3, \beta=0$. Thus $\bar{x} \in\left\langle x, G_{2}\right\rangle$.

We now exploit the fact that $\beta=0$.

LeMan 4.4. Suppose that $c(2 k-3)=\bar{c}(2 k-3)=\beta=0$. Then the equations of Lemma 3.4 take the form

$$
\delta(k)=\alpha^{k-2} \bar{a}(k)
$$

and, for $N+2>k$,

(6) $\frac{F(a, k, N+2)}{a(k)}+\frac{A(N+2)}{a(k)}=\frac{G(\bar{a}, k, N+2)}{\bar{a}(k)}+\frac{B(N+2)}{\bar{a}(k)}$,

where

$$
\begin{gathered}
A(N+2)=\delta^{-2} \sum_{\omega=2}^{N^{\prime}} \sum_{j=\omega(k-2)}^{N} A(\omega, j) g(1, N-j, \omega), \\
B(N+2)=\left(\delta a^{k-2}\right)^{-1} \sum_{\omega=1}^{N^{\prime}-1} \sum_{j=\omega(k-2)}^{N-k+2} A(\omega, j) \\
\quad \times \sum_{i=k}^{N-j+2} \bar{a}(i) g(i-2, N-j, \omega), \\
g(1, N-j, \omega)
\end{gathered}
$$$$
=\sum_{q=1}^{N-j+1} \alpha^{q} \frac{S_{N-j+1}^{q}}{(N-j+1) !} \sum_{r=2}^{\omega+1} \delta^{r} \frac{S_{\omega+1}^{r}}{(\omega+1) !} 2 ! \delta_{r}^{2},
$$ 


$$
\begin{aligned}
g(i-2, N-j, \omega) & \\
= & \sum_{z=i-1}^{N-j+1} \alpha^{z} \frac{S_{N-j+1}^{z}}{(N-j+1) !}(i-1) ! \delta_{z}^{i-1} \sum_{u=1}^{\omega+1} \delta^{u} \frac{S_{\omega+1}^{u}}{(\omega+1) !},
\end{aligned}
$$

and $N^{\prime}=[N /(k-2)]$.

Proof. The left side of (6) is $L(N) /\left(a(k) \delta^{2}\right)$, where $L(N)$ is defined in Lemma 3.4. The sum $F(a, k, N+2)$ corresponds to that part of $L(N)$ where $\omega=1$.

As for the right side of (6), $G(\bar{a}, k, N+2)$ corresponds to $R_{1}(N)$ and $B(N+2)$ to $R_{2}(N)$. In evaluating $R_{2}(N)$ we have, at first,

$$
R_{2}(N)=\sum_{\omega=1}^{N^{\prime}} \sum_{j=\omega(k-2)}^{N} A(\omega, j) \sum_{i=k}^{N+5-k} \bar{a}(i) g(i-2, N-j, \omega) .
$$

The indices of summation here differ from those given in the defining sum for $B(N+2)$. But by Lemma 2.3,

$$
\begin{aligned}
g(i- & 2, N-j, \omega) \\
& =\sum_{z=i-1}^{N-j+1} \alpha^{z} \frac{S_{N-j+1}^{z}}{(N-j+1) !} \sum_{u=1}^{\omega+1} \delta^{u} \frac{S_{\omega+1}^{u}}{(\omega+1) !}(i-1) ! \delta_{z}^{i-1} .
\end{aligned}
$$

Thus, $N-j+1>i-1$. Using this fact one gets the displayed indices of summation for $B(N+2)$.

Applying Lemma 4.2 to Lemma 4.4 we get the initial case of an induction:

LEMMA 4.5. There is an integer $\lambda_{1}$, a sequence $q(0), \ldots, q\left(\lambda_{1}\right)$, an associated set of nonzero numbers $\left\{a_{i}(q(i))\right\}$, and a sequence $\{c(i): i>2(k-2)+2\}$ such that the system of equations of Lemma 4.4 is equivalent to the system

$$
\begin{gathered}
\delta a(k)=\alpha^{k-2} \bar{a}(k), \\
a_{i}(q(i))=\alpha^{q(i)-q(i-1)} \bar{a}_{i}(q(i)), \quad i=1, \ldots, \lambda_{1},
\end{gathered}
$$

and for $N+2 \geqslant 2(k-2)+2=k^{\prime}$,

$$
\begin{aligned}
F\left(c, k^{\prime}, N+2\right) & +A(N+2) / P\left(\lambda_{1}\right) \\
& =\alpha^{k^{\prime}-q} G\left(\bar{c}, k^{\prime}, N+2\right)+\alpha^{k-q} B(N+2) / \bar{P}\left(\lambda_{1}\right)
\end{aligned}
$$

where $q=q\left(\lambda_{1}\right)$. Furthermore, for $i<2(k-2)+1$,

$$
a(i)=\sum_{\nu=0}^{\lambda_{1}} P(\nu) \sigma(q(\nu)-2, i-2) .
$$

For $i>2(k-2)+1$, 


$$
a(i)=\sum_{i=0}^{\lambda_{1}} P(\nu) \sigma[q(\nu)-2, i-2]+P\left(\lambda_{1}\right) c(i) .
$$

To continue, given $q(0), \ldots, q(1)$ and $a_{0}(q(0)), \ldots, a_{i}(q(i))$ define the t-fold sum $T_{t}(m, y)$ by

$$
T_{t}(m, y)=\sum_{\nu_{1}=0}^{y} \cdots \sum_{v_{t}=0}^{y} P\left(\nu_{1}\right) \cdots P\left(\nu_{t}\right) \sigma[Q(t)-2 t, m],
$$

where $P(\nu)=a_{0}(q(0)) \cdots a_{\nu}(q(\nu))$ and $Q(t)=Q\left(\nu_{1}, \ldots, \nu_{t}\right)=q\left(\nu_{1}\right)$ $+\cdots+q\left(\nu_{t}\right)$. By convention let $\Sigma_{(v)}^{(y)},-(0)$ denote the $t$-fold sum with indices of summation $v_{j}=0, \ldots, y$ for $j=1, \ldots, t$.

The general step of the induction is given by

LEMMA 4.6. Given $r>1$ there is an integer $\lambda_{r}$ and a sequence $q(0), \ldots, q\left(\lambda_{r}\right)$ such that the system of equations of Lemma 4.5 is equivalent to the system

$$
\begin{gathered}
\alpha a(k)=\alpha^{k-2} \bar{a}(k), \\
a_{i}(q(i))=\alpha^{q(i)-q(i-1)} \bar{a}_{i}(q(i)), \quad i=1, \ldots, \lambda_{r},
\end{gathered}
$$

and for $N+2>(r+1)(k-2)+2$,

$$
\begin{aligned}
F\left(c, k^{\prime}, N+2\right) & +A(N+2) / P\left(\lambda_{1}\right) \\
& =\alpha^{k^{\prime}-q} G\left(\bar{c}, k^{\prime}, N+2\right)+\alpha^{k-q} B(N+2) /\left(\bar{P} \lambda_{1}\right),
\end{aligned}
$$

where $k^{\prime}=2(k-2)+2, q=q\left(\lambda_{1}\right)$, and $\{c(i)\}$ is the sequence given in Lemma 4.5. In addition, if $i<(r+1)(k-2)+1$ then

$$
a(i)=\sum_{j=1}^{r} \frac{T_{j}\left(i-2, \lambda_{r}\right)}{j !} .
$$

Finally, if $l<r$ then $q\left(\lambda_{l}+1\right)>(l+1)(k-2)+2$.

There are two steps to the proof of Lemma 4.6. First we employ (7) to compute $A(N+2)$ and $B(N+2)$ for the "next block"; the results are summarized in Lemma 4.7. Then, in Lemma 4.14, we "shift" the sequence $\{c(i)\}$; the shift gives us a system of equations which can be treated by Lemma 4.2.

LeMaA 4.7. The equations of Lemma 4.5 corresponding to $2(k-2)+2<N$ $+2<(r+2)(k-2)+1$ reduce to

$$
\begin{aligned}
F\left(c, k^{\prime}, N+2\right)-\sum_{i=2}^{r+1} \sum_{(\nu)_{i}-(0)}^{\left(\lambda_{1}\right)} \frac{P\left(\nu_{1}\right) \cdots P\left(\nu_{i}\right)}{i ! P\left(\lambda_{1}\right)} M(\alpha) \\
=\alpha^{k^{\prime}-q} G\left(\bar{c}, k^{\prime}, N+2\right)-\sum_{i=2}^{r+1} \sum_{(\nu)_{1}-(0)}^{\left(\lambda_{1}\right)} \frac{P^{*}\left(\nu_{1}\right) \cdots P^{*}\left(\nu_{i}\right)}{i ! P^{*}\left(\lambda_{1}\right)} M(\alpha),
\end{aligned}
$$


where $P^{*}(\nu)=\alpha^{q(v)-2} \bar{P}(\nu)$,

$$
\begin{aligned}
M(\alpha) & =M\left(\nu_{1}, \ldots, \nu_{i} ; \alpha\right) \\
& =\sum_{m=1}^{N+1-Q(i)+2 i} \frac{\alpha^{m}}{m !} \sigma[Q(i)-2 i+m, N+1],
\end{aligned}
$$

and $Q(i)=q\left(\nu_{1}\right)+\cdots+q\left(\nu_{i}\right)$.

Note. The equations of Lemma 4.7 corresponding to $2(k-2)+2 \leqslant N+$ $2<(r+1)(k-2)+1$ are redundant; they were reduced by the first $r$ steps of the induction. They are included in this $(r+1)$ st step for the sake of convenience.

There are several steps to the proof of Lemma 4.7. To start we compute $A(N+2)$ when $N+2<(r+2)(k-2)+1$. By Lemma 4.4,

$$
\delta^{2} A(N+2)=\sum_{\omega=2}^{r+1} \sum_{j=\omega(k-2)}^{N} A(\omega, j) g(1, N-j, \omega),
$$

for $N^{\prime}=[N /(k-2)]<r+1$. Next, the index of summation of $A(\omega, j)$ consists of those $\left(i_{1}, \ldots, i_{\omega}\right)$ with $i_{1}+\cdots+i_{\omega}=j+2 \omega$. Since $i_{\nu}>k$ for each $\nu$ we have

$$
\begin{aligned}
i_{1}+(\omega-1) k & <j+2 \omega<N+2 \omega \\
& <(r+2)(k-2)-1+2 \omega .
\end{aligned}
$$

That is, $i_{1}<(r+1)(k-2)+1$. Consequently, (7) can be used to compute $A(\omega, j)$ :

LEMMA 4.8. Suppose that $\omega<r+1$ and $j<(r+2)(k-2)+1$. Then

$$
A(\omega, j)=\sum_{i=\omega}^{r+1} \frac{\omega !}{i !} \delta_{i}^{\omega} T_{i}\left(j, \lambda_{r}\right)
$$

Proof. We have

$$
A(\omega, j)=\sum a\left(i_{1}\right) \cdots a\left(i_{\omega}\right)
$$

where $i_{1}+\cdots+i_{\omega}=j+2 \omega$. By (4)

$$
a(i)=\sum_{t>1} \frac{T_{t}\left(i-2, \lambda_{r}\right)}{t !}
$$

for $T_{t}\left(i-2, \lambda_{l}\right)=0$ for $t>r+1$. Setting this expression in the summatory function and summing on $i_{1}, \ldots, i_{\omega}$ one gets

$$
A(\omega, j)=\sum_{t_{1}>1} \cdots \sum_{t_{\omega}>1} \frac{T_{t_{1}+\cdots+t_{\omega}}\left(j, \lambda_{r}\right)}{t_{1} ! \cdots t_{\omega} !} .
$$

To complete the proof sum on those $t_{1}, \ldots, t_{\omega}$ with $t_{1}+\cdots+t_{\omega}=i$. 
LEMMA 4.9. Let $t_{0}=\max \{2, t-1\}$ and

$$
L_{l}(i)=2 ! \delta_{\imath}^{2} \sum_{\omega=t_{0}}^{i} \frac{S_{\omega+1}^{t}}{(\omega+1)} \frac{\delta_{i}^{\omega}}{i !} .
$$

Then for $N+2<(r+2)(k-2)+1$,

$$
A(N+2)=\sum_{i=2}^{r+1} \sum_{(\nu)_{i}-(0)}^{\left(\lambda_{1}\right)} P\left(\nu_{1}\right) \cdots P\left(\nu_{i}\right) M(\alpha) \sum_{i=2}^{i+1} \delta^{t-2} L_{t}(i),
$$

where $M(\alpha)$ is defined as in Lemma 4.7.

This follows from (8), Lemma 4.8 and Lemma 4.4.

Going on to the computation of $B(N+2)$ we have

Leman 4.10. Suppose that $(N+2)<(r+2)(k-2)+1$. Then

$$
\begin{aligned}
& \sum_{i=k}^{N-j+2} \bar{a}(i) g(i-2, N-j, \omega) \\
&=\sum_{i=1}^{r} \frac{1}{t !} \sum_{(\nu)_{1}-(0)}^{\left(\lambda_{1}\right)} \bar{P}\left(\nu_{1}\right) \cdots \bar{P}\left(\nu_{t}\right) M_{1}(\alpha)\left(\begin{array}{c}
\delta \\
\omega+1
\end{array}\right),
\end{aligned}
$$

where

$$
M_{1}(\alpha)=\sum_{z=Q(t)-2 t+1}^{N-j+1} \alpha^{z} \frac{S_{N+1-j}^{z}}{(N+1-j) !} \frac{z !}{(z-Q(t)+2 t) !} .
$$

This follows from Lemma 4.4, (7), and Lemma 2.1.

LEMMa 4.11. Set

$$
\begin{aligned}
& Q(t, u)=q\left(\nu_{1}\right)+\cdots+q\left(\nu_{t}\right) \\
& +q\left(\tau_{1}\right)+\cdots+q\left(\tau_{u}\right)-2 t-2 u, \\
& P(t, u)=\alpha^{Q(t, u)} \bar{P}\left(\nu_{1}\right) \cdots \bar{P}\left(\nu_{t}\right) \bar{P}\left(\tau_{1}\right) \cdots \bar{P}\left(\tau_{u}\right), \\
& S(\delta)=\sum_{\omega=1}^{u} \frac{\omega !}{\delta^{u}}\left(\begin{array}{c}
\delta \\
\omega+1
\end{array}\right) \aleph_{u}^{\omega},
\end{aligned}
$$

and

$$
M_{2}(\alpha)=\sum_{m=1}^{N+1-Q(t, u)} \frac{\alpha^{m}}{m !} \sigma[m+Q(t, u), N+1]
$$

Then 


$$
\delta \alpha^{k-2} B(N+2)=\sum_{\substack{u>1<>1 \\ u+t<r+1}} \frac{1}{u ! t !} \sum_{(v)_{t}-(0)}^{\left(\lambda_{1}\right)} \sum_{(\tau)_{u}-(0)}^{\left(\lambda_{r}\right)} P(t, u) S(\delta) M_{2}(\alpha) .
$$

This follows from Lemmas 4.4, 4.10, 4.8 and the fact that $\delta P(\nu)=$ $\alpha^{q(\nu)-2} \bar{P}(\nu)$.

Rearranging the quantities in Lemma 4.11 one gets

LEMMA 4.12. Let

$$
R_{m}(i)=\sum_{\substack{u=1-m \\(u>1)}}^{i-1} \frac{1}{(i-u) !} \sum_{\substack{\omega-u+m-1 \\(\omega>1)}}^{u} \frac{S_{\omega+1}^{m+u}}{\omega+1} \frac{S_{u}^{\omega}}{u !}
$$

Let $M(\alpha)$ and $P^{*}(\nu)$ be defined as in Lemma 4.7. Then

$$
\alpha^{k-2} B(N+2)=\sum_{i=2}^{r+1} \sum_{(\nu)_{i}-(0)}^{\left(\lambda_{1}\right)} P^{*}\left(\nu_{1}\right) \cdots P^{*}\left(\nu_{i}\right) M(\alpha) \sum_{m=2-i}^{1} \delta^{m-1} R_{m}(i) .
$$

Lemama 4.13. Suppose that

$$
F+A(N+2) / P\left(\lambda_{1}\right)=G+\alpha^{k-q} B(N+2) / \bar{P}\left(\lambda_{1}\right)
$$

where $q=q\left(\lambda_{1}\right)$. Then

$$
\begin{aligned}
F+\sum_{i=2}^{r+1} \sum_{(\nu)_{i}-(0)}^{\left(\lambda_{1}\right)} \frac{P\left(\nu_{1}\right) \cdots P\left(\nu_{i}\right)}{P\left(\lambda_{1}\right)} M(\alpha)\left(L_{2}(i)-R_{2-i}(i)\right) \\
=G+\sum_{i=2}^{r+1} \sum_{(\nu)_{i}-(0)}^{\left(\lambda_{1}\right)} \frac{P^{*}\left(\nu_{1}\right) \cdots P^{*}\left(\nu_{i}\right)}{P^{*}\left(\lambda_{1}\right)} M(\alpha)\left(R_{1}(i)-L_{i+1}(i)\right) .
\end{aligned}
$$

Proof. Fix $i,\left(v_{1}, \ldots, v_{i}\right)$ and $t$ in the equation for $A(N+2)$ given in Lemma 4.9. Suppose also that $3<t<i$. Then

$$
\frac{P\left(\nu_{1}\right) \cdots P\left(\nu_{i}\right)}{P\left(\lambda_{1}\right)} \delta^{t-2} L_{t}(i)=\delta^{t-i-1} \frac{P^{*}\left(\nu_{1}\right) \cdots P^{*}\left(\nu_{i}\right)}{P^{*}\left(\lambda_{1}\right)} L_{t}(i) .
$$

Next, by Lemma 2.2, $L_{t}(i)=R_{t-i}(i)$ when $3<t<i$. Thus the equation of the hypothesis of Lemma 4.13 reduces to 


$$
\begin{aligned}
F+\sum_{i=2}^{r+1} \sum_{(\nu)_{i}-(0)}^{\left(\lambda_{1}\right)} \frac{P\left(\nu_{1}\right) \cdots P\left(\nu_{i}\right)}{P\left(\lambda_{1}\right)}\left(L_{2}(i)+\delta^{i-1} L_{i+1}(i)\right) M(\alpha) \\
=G+\sum_{i=2}^{r+1} \sum_{(\nu)_{1}-(0)}^{\left(\lambda_{1}\right)} \frac{P^{*}\left(\nu_{i}\right) \cdots P^{*}\left(\nu_{i}\right)}{P^{*}\left(\lambda_{1}\right)}\left(\delta^{1-i} R_{2-i}(i)-R_{1}(i)\right) M(\alpha) .
\end{aligned}
$$

To complete the proof transpose the powers of $\delta$.

Lemma 4.7 follows from Lemma 4.13 for, by Lemma 2.2,

$$
L_{2}(i)-R_{2-i}(i)=R_{1}(i)-L_{i+1}(i)=-1 / i ! .
$$

As mentioned, we need to shift the sequence $\{c(i)\}$ :

LEMMA 4.14. Let, for $i<(r+2)(k-2)+1$,

$$
c(i)=e(i)+\sum_{j=2}^{r+1} \frac{1}{j !} \frac{T_{j}\left(i-2, \lambda_{r}\right)}{P\left(\lambda_{1}\right)} .
$$

Then the equations of Lemma 4.7 reduce to

$$
F\left(e, k^{\prime}, N+2\right)=\alpha^{k^{\prime}-q} G\left(\bar{e}, k^{\prime}, N+2\right) .
$$

In addition, for $i>(r+1)(k-2)+2$,

$$
\begin{aligned}
a(i)= & \sum_{\nu=0}^{\lambda_{1}} P(\nu) \sigma[q(\nu)-2, i-2] \\
& +\sum_{j=2}^{r+1} \frac{1}{j !} T_{j}\left(i-2, \lambda_{r}\right)+P\left(\lambda_{1}\right) e(i) .
\end{aligned}
$$

The definition of $c(i)$ given above is consistent with the inductive definition of $c(i)$ for $i<(r+1)(k-2)+1$. For if $i<(l+1)(k-2)+1$, where $l<$ $r$, then

$$
c(i)=e(i)+\sum_{j=2}^{l} \sum_{(v)_{j}-(0)}^{\left(\lambda_{1}\right)} \frac{P\left(\nu_{1}\right) \cdots P\left(\nu_{i}\right)}{j ! P\left(\lambda_{1}\right)} \sigma[Q(j)-2 j, i-2] .
$$

Next, the upper limits of summation here can be increased to $r+1$ and $\left(\lambda_{r}\right)$. If, say, $\nu_{1}>\lambda_{l+1}$, then

$$
q\left(\nu_{1}\right)>q\left(\lambda_{l}+1\right)>(l+1)(k-2)+2,
$$

and

$$
Q(j)-2 j>(l+1)(k-2)+2+(j-1) k-2 j>i-2 .
$$

Consequently, the corresponding Stirling number is zero.

The proof of Lemma 3.14 follows from (2.8) and Lemma 2.1.

We now apply Lemma 4.1 and then Lemma 4.2 to the equation $F\left(e, k^{\prime}, N\right.$ $+2)=\alpha^{k-q^{\prime}} G\left(e, k^{\prime}, N+2\right)$ to get a sequence $q\left(\lambda_{1}+1\right), \ldots, q\left(\lambda_{r}\right), q\left(\lambda_{r}+\right.$ 1), .., $q\left(\lambda_{r+1}\right)$, the corresponding equations, and the analogues of (7). This 
completes the proof of Lemma 4.6. Lemma 4.6, in turn, gives us parts (i) and (ii) of Theorem 2.

The fact that $q(0), \ldots, q\left(\lambda_{1}\right)$ is a sequence of group invariants follows from the implicit applications of Lemma 4.1 in the proof of Lemma 4.3. The invariance of $q\left(\lambda_{1}+1\right), \ldots, q(\lambda)$ follows from the uniqueness of the subgroup $H$ mentioned after the proof of Lemma 4.3.

The power equations of Theorem 2 were proved in $[4, p$. 365] for the $|G|>p^{p+1}$ case; the lemmas used there in the derivation did not depend on the size of $k$ relative to $n$. The fact that the equations in (iii) of Theorem 2 hold for $|G|<p^{p}$ follows from the regularity of $G$. The equations of the corollary to Theorem 2 are arrived at by examining the cases mentioned.

Theorem 3 is Lemma 38 of [4].

\section{BIBLIOGRAPHY}

1. N. Blackburn, On a special class of p-groups, Acta Math. 100 (1958), 45-92. MR 21 \# 1349.

2. C. L. Chang, On metabelian p-groups of maximal class, Dissertation, Univ. of California, Los Angeles, 1971.

3. B. Huppert, Endliche Gruppen, Die Grundlehren der math. Wissenschaften, Band 134, Springer-Verlag, Berlin and New York, 1967. MR 37 \#302.

4. R. J. Miech, Metabelian p-groups of maximal class, Trans. Amer. Math. Soc. 152 (1970), 331-373.

5. G. Szekeres, Metabelian groups with two generators, Proc. Internat. Conf. Theory of Groups, Gordon and Breach, New York, 1967, pp. 323-346.

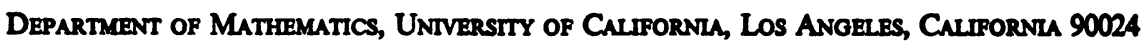

